\title{
High-Temperature Heat Capacity of Lanthanide Cuprates
}

\section{Liubov T. Denisova, Liubov G. Chumilina, Viktor M. Denisov* and Sergey D. Kirik* Siberian Federal University 79 Svobodny, Krasnoyarsk, 660041 Russia}

Received 12.01.2015, received in revised form 02.02.2015, accepted 03.03.2015

This paper presents data on the heat capacity of lanthanide cuprates obtained in the temperature range $354-877 \mathrm{~K}$. The thermodynamic functions of the solid oxide compound have been calculated using the experimental data.

Keywords: heat capacity, enthalpy, entropy, oxides of lanthanum and copper.

\section{Теплоемкость купрата лантана}

в области 354-877 К

Л.Т. Денисова, Л.Г. Чумилина, В.М. Денисов, С.Д. Кирик

Сибирский федеральный университет Россия, 660041, Красноярск, пр. Свободный, 79

Получены данные по теплоемкости $\mathrm{La}_{2} \mathrm{Cu}_{2} \mathrm{O}_{5}$ в интервале температур 354-877 К. По экспериментальным данным рассчитаны термодинамические функиии твердого оксидного соединения.

Ключевые слова: теплоемкость, энтальпия, энтропия, оксиды лантана и меди.

(C) Siberian Federal University. All rights reserved

* Corresponding author E-mail address: antluba@mail.ru 


\section{Введение}

Несмотря на то что соединение $\mathrm{La}_{2} \mathrm{Cu}_{2} \mathrm{O}_{5}$ длительное время привлекает внимание исследователей [1-11], сведения о высокотемпературной теплоемкости в литературе отсутствуют. Данные о равновесной диаграмме состояния системы $\mathrm{La}_{2} \mathrm{O}_{3}-\mathrm{CuO}$ приведены в работах $[1,9,12$, 13]. По данным $[1,12]$ она характеризуется наличием двух соединений $-\mathrm{La}_{2} \mathrm{CuO}_{4}$ и $\mathrm{La}_{2} \mathrm{Cu}_{2} \mathrm{O}_{5}$. В то же время согласно $[9,13]$ в этой системе образуются три соединения: $\mathrm{La}_{2} \mathrm{CuO}_{4}, \mathrm{La}_{8} \mathrm{Cu}_{7} \mathrm{O}_{19}$ и $\mathrm{La}_{2} \mathrm{Cu}_{2} \mathrm{O}_{5}$. Соединение $\mathrm{La}_{2} \mathrm{Cu}_{2} \mathrm{O}_{5}$ является членом гомологического ряда $\mathrm{La}_{2 \mathrm{n}+2} \mathrm{Cu}_{\mathrm{n}+4} \mathrm{O}_{4 \mathrm{n}+7}$ при $\mathrm{n}=2[2]$.

Целью настоящей работы стало измерение высокотемпературной теплоемкости и расчета по этим данным термодинамических свойств $\mathrm{La}_{2} \mathrm{Cu}_{2} \mathrm{O}_{5}$.

\section{Результаты экспериментов и их обсуждение}

Принимая во внимание, что $\mathrm{La}_{2} \mathrm{Cu}_{2} \mathrm{O}_{5}$ плавится инконгруэнтно $[9,13]$, получали его твердофазным синтезом. В данной технологии получения этого соединения существует противоречие. Согласно [6] $\mathrm{La}_{2} \mathrm{Cu}_{2} \mathrm{O}_{5}$ не может быть получен при $\mathrm{T}=1373$ К реакцией между $\mathrm{La}_{2} \mathrm{CuO}_{4}$ и $\mathrm{CuO}$, в то время как, по данным [14], по названной реакции соединение может быть получено при $\mathrm{T}=1275$ К. В обоих случаях неизвестно время синтеза, количество помолов и т.д. По нашим данным, при синтезе по указанной выше реакции при температуре 1273 К в течение 35 ч (с промежуточными перетираниями через каждые 5 ч и последующими прессованиями) образуется $\mathrm{La}_{2} \mathrm{Cu}_{2} \mathrm{O}_{5}$, не содержащий исходных продуктов. Контроль получаемых образцов проводили с использованием рентгенофазового анализа. На рентгенограммах, полученных на приборе X'Pert Pro фирмы Panalytical (Нидерланды), присутствовали только рефлексы, отвечающие соединению $\mathrm{La}_{2} \mathrm{Cu}_{2} \mathrm{O}_{5}$. Полученные нами параметры структуры в сравнении с данными других авторов приведены в табл. 1.

Из данных, представленных в табл. 1, следует, что наши результаты в целом согласуются с имеющимися сведениями о параметрах структуры $\mathrm{La}_{2} \mathrm{Cu}_{2} \mathrm{O}_{5}$. При этом лучшее согласие наблюдается с данными авторов работы $[6,7]$.

Измерение теплоемкости проводили в платиновых тиглях на приборе STA 449 C Jupiter (NETZSCH). Методика экспериментов с помощью дифференциальной сканирующей калориметрии описана нами ранее $[15,16]$.

Таблица 1. Параметры структуры $\mathrm{La}_{2} \mathrm{Cu}_{2} \mathrm{O}_{5}$

\begin{tabular}{|c|c|c|c|c|}
\hline $\mathrm{a}, \AA$ & $\mathrm{b}, \AA$ & $\mathrm{c}, \AA$ & $\beta$, град & Источник \\
\hline $13,86(1)$ & 3,74 & $27,99(1)$ & $106,3(1)$ & {$[5]$} \\
$13,8640(14)$ & $3,7469(3)$ & $27,943(3)$ & $106,06(2)$ & {$[6]$} \\
$13,8640(14)$ & $3,7469(3)$ & $27,943(3)$ & $106,06(2)$ & {$[7]$} \\
13,82 & $3,758(3)$ & $28,030(3)$ & $106,304(4)$ & {$[8]$} \\
& & 27,964 & 106,06 & {$[9]$} \\
13,874 & 3,752 & 27,956 & 106,05 & {$[10]$} \\
$13,893(9)$ & $3,754(2)$ & $27,587(11)$ & $102,84(7)$ & {$[11]$} \\
13,8640 & 3,7469 & 27,9430 & 106,06 & Наши данные \\
\hline
\end{tabular}


На рис. 1 приведена температурная зависимость теплоемкости $\mathrm{La}_{2} \mathrm{Cu}_{2} \mathrm{O}_{5}$. Видно, что значения $\mathrm{C}_{\mathrm{p}}$ закономерно увеличиваются с ростом температуры, а на кривой $\mathrm{C}_{\mathrm{p}}=\mathrm{f}(\mathrm{T})$ нет различного рода экстремумов. Полученные данные могут быть описаны уравнением (Дж/(моль))

$$
\mathrm{C}_{\mathrm{p}}=205,25+36,80 \cdot 10^{-3} \mathrm{~T}-9,14 \cdot 10^{5} \mathrm{~T}^{-2} .
$$

Наличие зависимости $\mathrm{C}_{\mathrm{p}}=\mathrm{f}(\mathrm{T})$ позволяет по известным термодинамическим уравнениям рассчитать изменение энтальпии $\mathrm{H}_{\mathrm{T}}^{0}-\mathrm{H}_{354}^{0}$ и энтропии $\mathrm{S}_{\mathrm{T}}^{0}-\mathrm{S}_{354}^{0}$. Результаты расчета приведены в табл. 2.

На рис. 1 приведены также значения $\mathrm{C}_{\mathrm{p}}$, рассчитанные в модели Дебая [17]. Использовали при этом значение характеристической температуры Дебая $\Theta_{\mathrm{D}}=425 \mathrm{~K}$, полученное на основании величин $\mathrm{C}_{\mathrm{p}}$ при низкой температуре. Заметим, что в модели Дебая рассчитывают $\mathrm{C}_{\mathrm{V}}$, а не $\mathrm{C}_{\mathrm{p}}$. Существует много уравнений, позволяющих рассчитать разность $\mathrm{C}_{\mathrm{p}}-\mathrm{C}_{\mathrm{V}}[17-19]$. Тем не

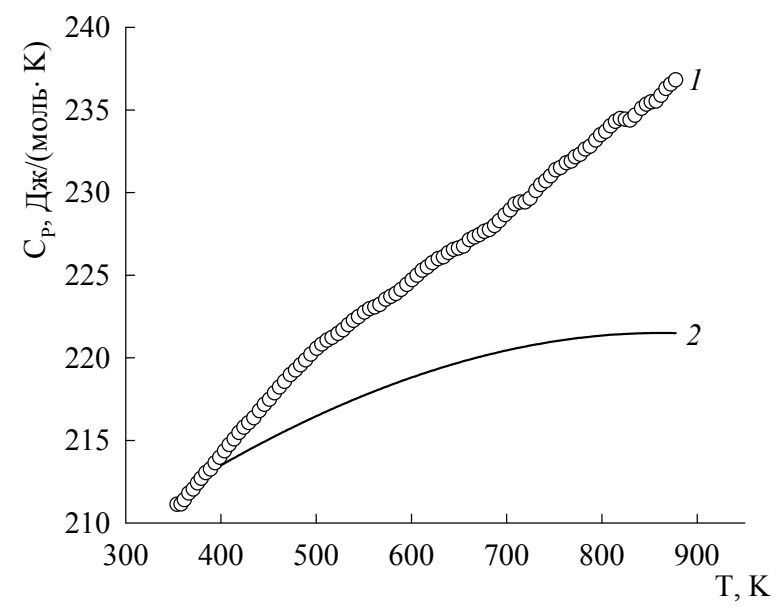

Рис. 1. Температурная зависимость теплоемкости $\mathrm{La}_{2} \mathrm{Cu}_{2} \mathrm{O}_{5}: 1$ - экспериментальные данные; 2 - расчет по модели Дебая

Таблица 2. Термодинамические свойства $\mathrm{La}_{2} \mathrm{Cu}_{2} \mathrm{O}_{5}$

\begin{tabular}{|c|c|c|c|}
\hline Т, $\mathrm{K}$ & $\begin{array}{c}\mathrm{C}_{\mathrm{p}}, \\
\text { Дж/(мольК) }\end{array}$ & $\begin{array}{c}\mathrm{H}_{\mathrm{T}}^{0}-\mathrm{H}_{354}^{0}, \\
\text { кДж/моль }\end{array}$ & $\begin{array}{c}\mathrm{S}_{\mathrm{T}}^{0}-\mathrm{S}_{354}^{0}, \\
\text { Дж/(мольК) }\end{array}$ \\
\hline 354 & 210,99 & - & - \\
400 & 214,26 & 9,78 & 25,98 \\
450 & 217,30 & 20,57 & 51,39 \\
500 & 220,00 & 31,51 & 74,43 \\
550 & 222,47 & 42,57 & 95,51 \\
600 & 224,79 & 53,75 & 115,0 \\
650 & 227,01 & 65,05 & 133,1 \\
700 & 229,14 & 76,45 & 150.0 \\
750 & 231,23 & 87,96 & 165,8 \\
800 & 233,26 & 99,57 & 190,8 \\
850 & 235,27 & 111,2 & 195,0 \\
\hline
\end{tabular}


менее для того чтобы ими воспользоваться, требуются дополнительные данные. Так, например, для уравнения [19]

$$
\mathrm{C}_{\mathrm{p}}=\mathrm{C}_{\mathrm{V}}\left(1+3 \gamma_{\mathrm{G}} \alpha \mathrm{T}\right)
$$

нужны сведения по температурному коэффициенту линейного расширения $\alpha$ и постоянной Грюнайзена $\gamma_{\mathrm{G}}$. Для соединения $\mathrm{La}_{2} \mathrm{Cu}_{2} \mathrm{O}_{5}$ такие данные нами не найдены. Кроме того, есть сведения о том, что теория теплоемкости Дебая для ряда оксидных кристаллов имеет приближенный характер, а коэффициент Грюнайзена для них сложным образом зависит от температуры [20]. Поэтому можно предположить, что в первом приближении $\mathrm{C}_{\mathrm{p}}$ и $\mathrm{C}_{\mathrm{V}}$ для $\mathrm{La}_{2} \mathrm{Cu}_{2} \mathrm{O}_{5}$ не очень отличаются между собой. Это позволит сравнивать рассчитанное значение в модели Дебая с экспериментом. Из рис. 1 следует, что начиная с температуры $\sim 400$ К наблюдается некоторое различие рассчитанных и экспериментальных значений $\mathrm{C}_{\mathrm{p}}$, причем с ростом температуры это различие увеличивается.

Анализ этих результатов проведем подобно [19] в предположении, что избыточная теплоемкость в анализируемом интервале температур описывается соотношением

$$
\Delta \mathrm{C}=\mathrm{R}\left(\frac{\Theta_{\mathrm{E}}}{\mathrm{T}}\right)^{2} \exp \left(-\frac{\Theta_{\mathrm{E}}}{\mathrm{T}}\right),
$$

где $\Theta_{\mathrm{E}}$ - характеристическая температура Эйнштейна. Представление полученных результатов в координатах $\ln \left(\Delta \mathrm{CT}^{2}\right)-1 / \mathrm{T}$ дает прямую линию (рис. 2) со значением коэффициента корреляции $\mathrm{r}=0,9982$. Соблюдение вкладов типа (3), по мнению авторов работы [19], означает, что колебательный спектр соединения $\mathrm{La}_{2} \mathrm{Cu}_{2} \mathrm{O}_{5}$ имеет локализованную оптическую моду, которая образуется в упорядоченных системах, если массы атомов компонентов существенно различаются.

Ранее нами была показана связь между составом оксидных соединений и их удельной теплоемкостью [22]. Из рис. 3 следует, что она наблюдается и для системы $\mathrm{La}_{2} \mathrm{O}_{3}-\mathrm{CuO}$. Это позволяет оценить значение $\mathrm{C}_{\mathrm{p}}^{\mathrm{o}}$ для соединения $\mathrm{La}_{8} \mathrm{Cu}_{7} \mathrm{O}_{19}$, для которого экспериментальные значения отсутствуют. Значение $\mathrm{C}_{\mathrm{p}}^{\mathrm{o}}\left(\mathrm{La}_{8} \mathrm{Cu}_{7} \mathrm{O}_{19}\right)$ равно 0,40(7) Дж/(г·K).

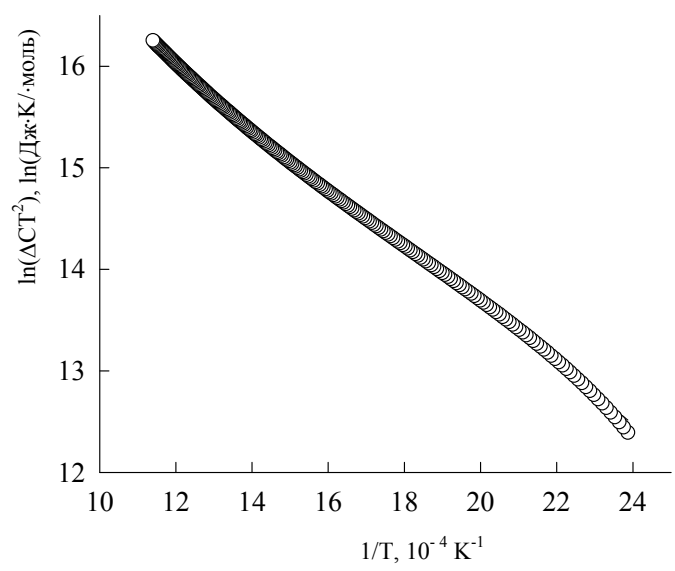

Рис. 2. Аппроксимация избыточной теплоемкости функцией Эйнштейна при $\mathrm{T}<<\Theta_{\mathrm{E}}$ 


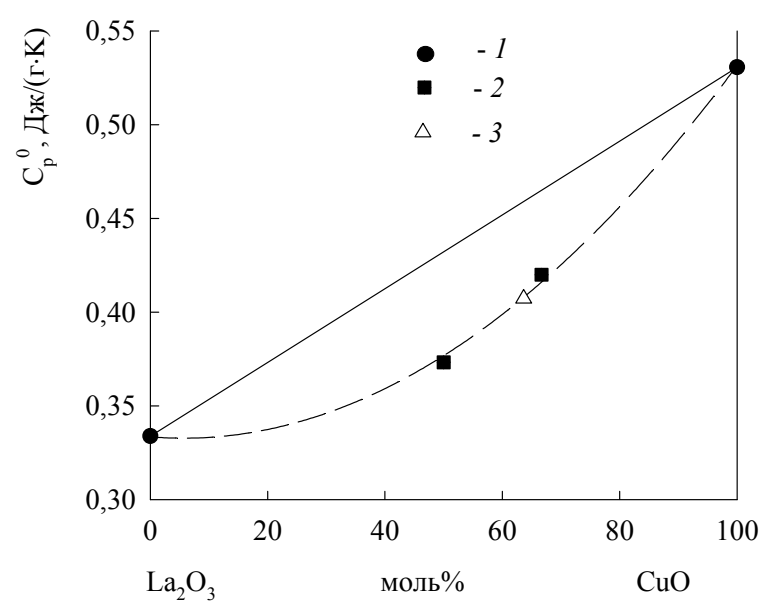

Рис. 3. Связь состава и удельных значений теплоемкости системы $\mathrm{CuO}-\mathrm{La}_{2} \mathrm{O}_{3}: 1-[23]$; 2 - наши данные; 3 - оценочные значения

\section{Список литературы}

1. Maljuk A.N., Emel'chenko G.A., Zver'kova I.I. et al. Crystallization regions of the $\mathrm{La}_{2} \mathrm{Cu}_{2} \mathrm{O}_{5}$ and $\mathrm{La}_{1-\mathrm{x}} \mathrm{Sr}_{\mathrm{x}} \mathrm{CuO}_{2,5-\delta}$ phases un the $\mathrm{La}_{2} \mathrm{O}_{3}-\mathrm{SrO}-\mathrm{CuO}$ system // Supercond. Sci. Technol. 1994. V. 7. P. 596-601.

2. Norrestam R., Nygren M., Bovin J.-O. New intermediate phases of the composition $\mathrm{La}_{2 \mathrm{n}+2} \mathrm{Cu}_{\mathrm{n}+4} \mathrm{O}_{4 \mathrm{n}+7}$ in the $\mathrm{La}-\mathrm{Cu}-\mathrm{O}$ system: the crystal structure and thermal stability of $\mathrm{La}_{2} \mathrm{Cu}_{2} \mathrm{O}_{5} / /$ Angew. Chem. Int. Ed. Engl. 1991. V. 30. № 7. P. 864-866.

3. Sekar C., Watanabe T., Matsuda A. et al. Effect of silver addition on structure and electrical properties of the spin ladder compound $\mathrm{La}_{2} \mathrm{Cu}_{2} \mathrm{O}_{5}$ single crystals // J. Cryst. Growth. 2001.V. 233. P. 466-472.

4. Kudo K., Noji T., Koike Y. et al. Thermal conductivity of the four-leg spin-ladder system $\mathrm{La}_{2} \mathrm{Cu}_{2} \mathrm{O}_{5}$ single crystal // J. Low Temp. Phys. 2003. V. 131. № 3/4. P. 725-729.

5. Golosovsky I.V., Gukasov A.G., Polyakov V.A. et al. Magnetic structure of lanthanum copper oxide $\mathrm{La}_{2} \mathrm{Cu}_{2} \mathrm{O}_{5} / /$ J. Phys.: Condens. Mater. 1999. V. 11. P. 6959-6967.

6. Cava R.J., Siegrist T., Hessen B. et al. A new type homologous series in the $\mathrm{La}-\mathrm{Cu}-\mathrm{O}$ system // Physica C. 1991. V. 177. P. 115-121.

7. Cava R.J., Siegrist T., Hessen B. et al. A new homologous series of lanthanum copper oxides // J. Solid State Chem. 1991. V. 94. P. 170-184.

8. Ionov A.M., Maljuk A.N. LEED and spectroscopic studies of the atomic and electronic structure of $\mathrm{La}_{2} \mathrm{Cu}_{2} \mathrm{O}_{5} / /$ Surface Sci. 1997. V. 372. P. 323-328.

9. Sekar C., Watanabe T., Matsuda A. Crystal growth and characterization of the 4-leg ladder compound $\mathrm{La}_{2} \mathrm{Cu}_{2} \mathrm{O}_{5} / /$ J. Cryst. Growth. 2000. V. 212. P. 142-147.

10. Sekar C., Watanabe T., Matsuda A. et al. Crystal growth and transport properties of 4-leg spin-ladder compound $\mathrm{La}_{2} \mathrm{Cu}_{2} \mathrm{O}_{5}$ : occurrence of insulator-metal transition // Inter. J. Inorg. Mater. 2001. V. 3. P. 1201-1203.

11. Norrestam R., Nygren M., Bovin J. -O.-Neue Zwischenphasen der Zusammensetzung $\mathrm{La}_{2 \mathrm{n}+2} \mathrm{Cu}_{\mathrm{n}+4} \mathrm{O}_{4 \mathrm{n}+7} \mathrm{im} \mathrm{La}-\mathrm{Cu}-\mathrm{O}-$ System; Kristallstruktur und thermische Stabilität von $\mathrm{La}_{2} \mathrm{Cu}_{2} \mathrm{O}_{5} / /$ Angew. Chem. 1991. B. 103. № 7. S. 891-893. 
12. Maljuk A.N., Kulakov A.B., Emel'chenko G.A. Temperature dependence of the dissolution enthalpy of the $\mathrm{La}_{2} \mathrm{CuO}_{4}$ and $\mathrm{Nd}_{2} \mathrm{CuO}_{4}$ phases and complex formation in cuprate melts // J. Cryst. Growth. 1995. V. 151. P. 102-106.

13. Schüpp B., Sekar C., Gruner W. et al. Crystal structure investigations and thetmal behavior of the five-leg spin ladder compound $\mathrm{La}_{8} \mathrm{Cu}_{7} \mathrm{O}_{19} / /$ Z. Anorg. Alg. Chem. 2004. V. 630. P. 663-668.

14. Geny J., Meen J.K., Elthon D. Phase equilibria of the $\mathrm{La}_{2} \mathrm{O}_{3}-\mathrm{SrO}-\mathrm{CuO}$ system at $950{ }^{\circ} \mathrm{C}$ and $10 \mathrm{kbar} / /$ J. Am. Ceram. Soc. 1996. V. 79. № 12. P. 3083-3089.

15. Денисов В.М., Денисова Л.Т., Иртюго Л.А. и др. Теплофизические свойства монокристаллов $\mathrm{Bi}_{4} \mathrm{Ge}_{3} \mathrm{O}_{12} / /$ Физика тверд. тела. 2010. Т. 52. № 7. С. 1274-1277 [Denisov V.M., Denisova L.T., Irtyugo L.A. et al. Thermal physical properties of $\mathrm{Bi}_{4} \mathrm{Ge}_{3} \mathrm{O}_{12}$ single crystals / / Physics of the Solid State. 2010. V. 52 (7). P. 1362-1365].

16. Денисов В.М., Денисова Л.Т., Иртюго Л.А. и др. Высокотемпературная теплоемкость $\mathrm{BaFe}_{12} \mathrm{O}_{19}$ и $\mathrm{BaSc}_{0,5} \mathrm{Fe}_{11,5} \mathrm{O}_{19} / /$ Физика тверд. тела. 2012. T.54. № 12. С. 2229-2231. [Denisov V.M., Denisova L.T., Irtyugo L.A. et al. High-temperature heat capacity of $\mathrm{BaFe}_{12} \mathrm{O}_{19}$ and $\mathrm{BaSc}_{0.5} \mathrm{Fe}_{11.5} \mathrm{O}_{19} / /$ Physics of the Solid State. 2013. V. 55 (1). P. 240-242].

17. Скуратов С.М., Колесов В.П., Воробьев А.Ф. Термохимия. Ч. ІІ. М.: МГУ, 1966. 434 с. [Skuratov, S.M., Kolesov, V.P., and Vorob'ev, V.F. Termokhimiya (Thermochemistry). Moscow: Mosk. Gos. Univ., 1966, part 2. 434 p.]

18. Лившиц Б.Г., Крапошин В.С., Линецкий Я.Л. Физические свойства металлов и сплавов. М.: Металлургия, 1980. 320 c. [Livshic B.G., Kraposhin V.S., Lineckei Ya.L. Physical properties of metals and alloys. M. Metallurgia, 1980. 320 p.]

19. Прекул А.Ф., Казанцев В.А., Щеголихина Н.И. и др. Высокотемпературная теплоемкость квазикристалла $\mathrm{Al}_{63} \mathrm{Cu}_{25} \mathrm{Fe}_{12} / /$ Физика тверд. тела. 2008. Т. 50. № 11. С. 1933-1935. [Prekul A.F., Kazancev V.A., Schegolihina N.I. et al. High-temperature heat capacity of kvazikristalla // Fizika Tverdogo Tela. 2008. V. 50. № 11. P. 1933-1935. (In Russ.)]

20. Антюхов А.М., Пашинкин А.С., Моисеев Н.В. Теплоемкость гранатовых кристаллов в интервале 4,3-300 К // Третья Всесоюзн. конф. «Термодинамика и материаловедение полупроводников». М.: АН СССР, 1986. T. III. C. 162-163. [Antuhov A.M., Pashinkin A.S., Moiseev, N.V. Heat capacity of garnet chip in the range 4,3-300 K // Tret'ya Vsesoyuznaya konferentsiya «Termodinamika i materialovedenie poluprovodnikov». 1986. V. III. P. 162-163. (In Russ.)]

21. Денисов В.М., Иртюго Л.А., Денисова Л.Т. Высокотемпературная теплоемкость оксидов системы $\mathrm{GeO}_{2}-\mathrm{PbO} / /$ Физика тверд. тела. 2011. Т. 53. № 4. С. 642-646. [Denisov V.M., Irtyugo L.A., Denisova L.T. High-temperature heat capacity of oxides in the $\mathrm{GeO}_{2}-\mathrm{PbO}$ system // Physics of the Solid State. 2011. V. 53 (4). P. 689-693].

22. Leitner J., Chuchvalec P., Sedmidubský D. et al. Estimation of heat capacities of solid mixed oxides // Thermochem. Acta. 2003. V. 395. P. 27-46. 